S. Mitra, Department of Mathematics, S.B College, Magra, W.B., India. S. N. Mukhopadhyay, Department of Mathematics, University of Burdwan, Burdwan,W.B., India.

\title{
DERIVATES, APPROXIMATE DERIVATES AND POROSITY DERIVATES OF $n$-CONVEX FUNCTIONS
}

\begin{abstract}
It is shown that if $f$ is $n$-convex then the four $n$th order Peano derivates of $f$ are respectively equal to the corresponding $n$th order approximate Peano derivates and the porosity Peano derivates of $f$. It is further shown that the same result holds for the de la Vallée Poussin derivates, and the symmetric and unsymmetric Riemann derivates.
\end{abstract}

\section{Introduction}

It is well known that for a monotone function $f$ the four Dini derivates of $f$ are equal to the corresponding approximate derivates of $f$. This result which generalized a result of Khintchine, [5], appeared in [7]; the proof in [7] is for finite derivates but only minor modifications are necessary to allow for infinite derivates. The result remained unnoticed by many authors; Misik proved the same result in a different way in [8]. Since then the result has been attributed to Misik, (see [1, pp.154-155], [10, p.139], [11, p.286], [6, p. 652], [2, p. $526]$ etc.). In this paper we show that for an $n$-convex function $f$ the four $n$th order Peano derivates of $f$ are respectively equal to the corresponding $n$th order approximate Peano derivates and the porosity Peano derivates of $f$. It is further shown that the same result holds for the de la Vallée Poussin derivates, and the symmetric and unsymmetric Riemann derivates.

Key Words: Peano derivate, approximate derivate, de la Vallée Poussin derivate, porosity, $n$-convexity

Mathematical Reviews subject classification: 26A24

Received by the editors July 1, 1999 


\section{Definitions and Notations}

Let $E \subseteq \mathbb{R}$, let $I$ be any open interval, and let $\lambda(E, I)$ denote the length of the largest open sub-interval of $I$ that contains no point of $E$. For $x \in \mathbb{R}$. define the porosity of $E$ at $x$ from the right, and from the left as

$P^{+}(E, x)=\limsup _{h \rightarrow 0+} \frac{\lambda(E,(x, x+h))}{h}, \quad P^{-}(E, x)=\liminf _{h \rightarrow 0+} \frac{\lambda(E,(x-h, x))}{h}$,

respectively. The quantity $P(E, x)=\max \left\{P^{+}(E, x), P^{-}(E, x)\right\}$ is then called the porosity of $E$ at $x$. Details of porosity considerations can be found in [10].

Let $f: \mathbb{R} \mapsto \mathbb{R}$ and $x \in \mathbb{R}$; then the upper porosity limit of $f$ at $x$ on the right, or the upper right porosity limit of $f$ at $x$ is defined by:

$$
P-\limsup _{t \rightarrow x+} f(t)=\inf \{y ;\{\xi: f(\xi)<y\} \text { has porosity } 0 \text { on the right of } x\} .
$$

The other three porosity limits of $f$ at $x$ are defined analogously. If all four porosity limits of $f$ at $x$ are equal then the common value is called the porosity limit of $f$ at $x$, written $P-\lim _{t \rightarrow x} f(t)$.

The relations among ordinary, approximate and porosity upper right hand limits are as follows:

$$
P-\limsup _{t \rightarrow x+} f(t) \leq \lim \sup _{a p_{t \rightarrow x+}} f(t) \leq \limsup _{t \rightarrow x+} f(t) .
$$

The definition of $n$-convexity is as in [3].

The function $f$ is said to have a right Peano derivative at $x$ of order $k, k$ being a positive integer, if there exist real numbers $\alpha_{i}, 1 \leq i \leq k$, depending on $x$ and $f$ only, such that

$$
f(x+h)=f(x)+\sum_{i=1}^{k} \frac{\alpha_{i}}{i !} h^{i}+\frac{\epsilon_{k}(x, h ; f)}{k !} h^{k},
$$

where

$$
\lim _{h \rightarrow 0+} \epsilon_{k}(x, h ; f)=0 .
$$

The number $\alpha_{k}$ is called the right Peano derivative of $f$ at $x$ of order $k$, written $f_{(k)}^{+}(x)$. For convenience we take $\alpha_{0}=f(x)=f_{(0)}^{+}(x)$.

Suppose that $f_{(k)}^{+}(x)$ exists, and for $t \neq 0$ write

$$
\gamma_{(k+1)}^{+}(x, h ; f)=\gamma_{(k+1)}^{+}(x, h)=(k+1) !\left(\frac{f(x+h)-\sum_{i=0}^{k} \frac{f_{(i)}^{+}(x)}{i !} h^{i}}{h^{k+1}}\right) .
$$


The right upper Peano derivative of $f$ at $x$ of order $k+1$ is defined by

$$
\bar{f}_{(k+1)}^{+}(x)=\limsup _{h \rightarrow 0+} \gamma_{(k+1)}^{+}(x, h ; f) .
$$

The definitions of the right approximate Peano derivative, $f_{(k), \text { ap }}^{+}(x)$, and the derivate $\bar{f}_{(k+1) \text { ap }}^{+}(x)$, and the definitions of the right porosity Peano derivative $P f_{(k)}^{+}(x)$, and derivate $P \bar{f}_{(k+1)}^{+}(x)$ are obtained by taking the approximate limit and the porosity limit, respectively, at each stage beginning with (3). The definitions of other quantities, left, right, upper and lower versions of the above concepts are obtained analogously.

The first order Peano derivative, and derivates, are the usual first order derivative and Dini derivates respectively; the porosity versions of which are defined in a more general way in $[2,10]$.

The function $f$ is said to have a de la Vallée Poussin, or just dlVP derivative at $x$ of order $2 k, k$ being a positive integer, if there are real numbers $\beta_{0}, \beta_{2}, \ldots, \beta_{2 k}$ such that

$$
\frac{f(x+h)+f(x-h)}{2}=\sum_{i=0}^{k} \frac{\beta_{2 i}}{(2 i) !} h^{2 i}+\frac{\eta_{2 k}(x, h ; f)}{(2 k) !} h^{2 k},
$$

where

$$
\lim _{h \rightarrow 0} \eta_{2 k}(x, h ; f)=0 .
$$

In this case $\beta_{2 k}$ is called the dlVP derivative of $f$ at $x$ of order $2 k$, written $d_{2 k} f(x)$.

Suppose that $d_{2 k} f(x)$ exists and write

$$
\theta_{2 k+2}(x, h ; f)=\frac{(2 k+2) !}{h^{2 k+2}}\left(\frac{f(x+h)+f(x-h)}{2}-\sum_{i=0}^{k} \frac{d_{2 i} f(x)}{(2 i) !} h^{2 i}\right),
$$

and define the upper dlVP derivate of $f$ at $x$ of order $2 k+2$ as

$$
\bar{d}_{2 k+2} f(x)=\limsup _{h \rightarrow 0} \theta_{2 k+2}(x, h ; f) .
$$

Similarly if there are real numbers $\beta_{1}, \beta_{3}, \ldots, \beta_{2 k+1}$ such that

$$
\frac{f(x+h)-f(x-h)}{2}=\sum_{i=0}^{k} \frac{\beta_{2 i+1}}{(2 i+1) !} h^{2 i+1}+\frac{\eta_{2 k+1}(x, h ; f)}{(2 k+1) !} h^{2 k+1},
$$


where

$$
\lim _{h \rightarrow 0} \eta_{2 k+1}(x, h ; f)=0,
$$

then $\beta_{2 k+1}$ is called the dlVP derivative of $f$ at $x$ of order $2 k+1$, written $d_{2 k+1} f(x)$. The quantities $\theta_{2 k+3}(x, h ; f)$, and $\bar{d}_{2 k+3} f(x)$ are defined analogously.

The approximate dlVP derivative, $d_{m \text {,ap }} f(x)$ and derivate $\bar{d}_{m+2, \text { ap }} f(x)$, the porosity dlVP derivative $P d_{m} f(x)$, and derivate $P \bar{d}_{m+2} f(x)$ are defined in an analogous way by taking approximate and porosity limits respectively, starting from (4). The lower derivates of the above concepts are defined similarly.

The $k$ th order symmetric difference of $f$ at $x$ is defined by,

$$
\Delta_{k}(x, h ; f)=\sum_{i=0}^{k}(-1)^{k-i}\left(\begin{array}{c}
k \\
i
\end{array}\right) f\left(x+i h-\frac{k h}{2}\right) .
$$

The $k$ th order symmetric Riemann derivative and derivate $R D_{k} f(x)$ and $\overline{R D}_{k} f(x)$, their approximate analogues, $R D_{k, \text { ap }} f(x)$, and $\overline{R D}_{k, \text { ap }} f(x)$, and their porosity analogues $P R D_{k} f(x)$, and $P \overline{R D_{k}} f(x)$, are defined by taking the appropriate limits of $\Delta_{k}(x, h ; f) / h^{k}$ as $h \rightarrow 0$. The lower derivates of the above concepts are defined similarly.

Similarly considering the unsymmetric difference of $f$ at $x$,

$$
\Delta_{k}^{*}(x, h ; f)=\sum_{i=0}^{k}(-1)^{k-i}\left(\begin{array}{l}
k \\
i
\end{array}\right) f(x+i h),
$$

the $k$ th order unsymmetric Riemann derivative $R D_{k}^{*} f(x)$, derivates $\overline{R D}_{k}^{*} f(x)$ etc., and their approximate and porosity analogues, $R D_{k, \text { ap }}^{*} f(x), \overline{R D}_{k, \text { ap }}^{*,+} f(x)$, $P R D_{k}^{*} f(x), P \overline{R D}_{k}^{*,+} f(x)$, etc., are defined by taking appropriate limits of $\Delta_{k}^{*}(x, h ; f) / h^{k}$ as $h \rightarrow 0, \rightarrow 0+$.

\section{Properties of $n$-convex Functions}

The following properties of $n$-convex functions, which are obvious for the case $n=1$, i.e. for increasing functions, and which will be needed in the sequel, do not seem to be known before $[9,4,3]$.

Theorem 3.1. If $f: \mathbb{R} \mapsto \mathbb{R}$ is n-convex then for $t \geq 0$,

(i) $0 \leq t^{n} \frac{\gamma_{n}^{+}(x, t ; f)}{n !} \leq h^{n} \frac{\gamma_{n}^{+}(y, h ; f)}{n !}$ when $[x, x+t] \subseteq[y, y+h]$;

$$
0 \leq t^{n} \frac{\theta_{n}(x, t ; f)}{n !} \leq h^{n} \frac{\theta_{n}(y, h ; f)}{n !} \quad \text { when }\left[x-\frac{t}{2}, x+\frac{t}{2}\right] \subseteq\left[y-\frac{h}{2}, y+\frac{h}{2}\right] .
$$


Proof. For $n=1$ these are obvious since in that case $f$ is increasing.

Let $n=2$. Since $f$ is convex, the function $g=f_{+}^{\prime}$ is increasing and $f$ is an indefinite integral of $g$. So for any $\xi$

$$
g(\xi)-g(x) \leq g(\xi)-g(y) \text { if }[x, x+t] \subseteq[y, y+h],
$$

and

$$
g(x+\xi+t / 2)-g(x+\xi-t / 2) \leq g(y+\xi+h / 2)-g(y+\xi-h / 2),
$$

if $[x-t / 2, x+t / 2] \subseteq[y-h / 2, y+h / 2]$. Hence

$$
0 \leq \int_{x}^{x+t}(g(\xi)-g(x)) \mathrm{d} \xi \leq \int_{y}^{y+h}(g(\xi)-g(y)) \mathrm{d} \xi,
$$

and

$$
\begin{aligned}
0 \leq & \int_{-t / 2}^{t / 2}(g(x+\xi+t / 2)-g(x+\xi-t / 2)) \mathrm{d} \xi \\
& \leq \int_{-h / 2}^{h / 2}(g(y+\xi+h / 2)-g(y+\xi-h / 2)) \mathrm{d} \xi,
\end{aligned}
$$

which respectively give

$$
0 \leq t^{2} \frac{\gamma_{2}^{+}(x, t ; f)}{2} \leq h^{2} \frac{\gamma_{2}^{+}(y, h ; f)}{2}, \quad \text { and } \quad 0 \leq t^{2} \frac{\theta_{2}(x, t ; f)}{2} \leq h^{2} \frac{\theta_{2}(y, h ; f)}{2}
$$

proving (i) and (ii) in this case.

Suppose that they are true for $n=m$. Then for any $m$-convex function $\phi$

$$
0 \leq t^{m} \frac{\gamma_{m}^{+}(x, t ; \phi)}{m !} \leq h^{m} \frac{\gamma_{m}^{+}(y, h ; \phi)}{m !},
$$

whenever $[x, x+t] \subseteq[y, y+h]$, and

$$
0 \leq t^{m} \frac{\theta_{m}(x, t ; \phi)}{m !} \leq h^{m} \frac{\theta_{m}(y, h ; \phi)}{m !},
$$

whenever $[x-t / 2, x+t / 2] \subseteq[y-h / 2, y+h / 2]$.

Let $f$ be $m+1$-convex, then $\phi=f^{\prime}$ is $m$-convex and satisfies (5) and (6). We consider several cases. 
Case I Let $x=y$. Then from (5) and (6),

$$
0 \leq u^{m} \frac{\gamma_{m}^{+}(x, u ; \phi)}{m !} \text {, and } 0 \leq \frac{u^{m} \theta_{m}(x, u ; \phi)}{m !} \text {, for } t \leq u \leq h .
$$

Integrating these relations from $t$ to $h$ we get (5) and (6) with $m$ replaced by $m+1$, and $\phi$ replaced by $f$. Note that we have used the fact: $\phi_{(m-1)}^{+}=$ $\phi_{+}^{(m-1)}=f_{+}^{(m)}=f_{(m)}^{+}$. Thus we have proved (i) and (ii) in this case. For the other cases we tackle (i) and (ii) separately.

Case II (i) Let $x \neq y, x+t=y+h$; then $h=t+\alpha$, where $\alpha=x-y>$ 0 . Since for $u \geq 0,[x, x+u] \subset[y, x+u]=[y, y+u+\alpha]$, (5) gives that $0 \leq u^{m} \frac{\gamma_{m}^{+}(x, u ; \phi)}{m !} \leq(u+\alpha)^{m} \frac{\gamma_{m}^{+}(y, u+\alpha ; \phi)}{m !}$. Hence

$$
\begin{aligned}
0 \leq & \int_{0}^{t} u^{m} \frac{\gamma_{m}^{+}(x, u ; \phi)}{m !} \mathrm{d} u \\
& \leq \int_{0}^{t}(u+\alpha)^{m} \frac{\gamma_{m}^{+}(y, u+\alpha ; \phi)}{m !} \mathrm{d} u \leq \int_{0}^{h} v^{m} \frac{\gamma_{m}^{+}(y, v ; \phi)}{m !} \mathrm{d} v
\end{aligned}
$$

which gives (5) with $m$ and $\phi$ replaced by $m+1$ and $f$ respectively.

Case III (i) Let $y<x \leq x+t<y+h$. Set $k=t+\alpha$, where $\alpha=x-y>0$. Then $x+t=y+k$ and $[x, x+t] \subset[y, y+k] \subset[y, y+h]$. So by Case II (i)

$$
0 \leq t^{m+1} \frac{\gamma_{m+1}^{+}(x, t ; f)}{(m+1) !} \leq k^{m+1} \frac{\gamma_{m+1}^{+}(y, k ; f)}{(m+1) !}
$$

and by Case I

$$
k^{m+1} \frac{\gamma_{m+1}^{+}(y, k ; f)}{(m+1) !} \leq h^{m+1} \frac{\gamma_{m+1}^{+}(y, h ; f)}{(m+1) !}
$$

which together give (5) with $m$ and $\phi$ replaced by $m+1$ and $f$ respectively. This completes the proof of (i).

Case II (ii) Let $x \neq y, x+t / 2=y+h / 2$; then $h=t+2 \alpha$, where $\alpha=$ $x-y>0$. Since for $u \geq 0,[x-u / 2, x+u / 2] \subset[y-u / 2-\alpha, y+u / 2+\alpha]$ we have from (6) that

$$
0 \leq u^{m} \frac{\theta_{m}(x, u ; \phi)}{m !} \leq(u+2 \alpha)^{m} \frac{\theta_{m}(y, u+2 \alpha ; \phi)}{m !},
$$


and hence

$$
\begin{aligned}
0 \leq & \int_{0}^{t} u^{m} \frac{\theta_{m}(x, u ; \phi)}{m !} \mathrm{d} u \leq \\
& \int_{0}^{t}(u+2 \alpha)^{m} \frac{\theta_{m}(x, u+2 \alpha ; \phi)}{m !} \mathrm{d} u \leq \int_{0}^{h} v^{m} \frac{\theta_{m}(y, v ; \phi)}{m !} \mathrm{d} v,
\end{aligned}
$$

which gives (6) with $m$ and $\phi$ replaced by $m+1$ and $f$ respectively.

Case III (ii) Let $x \neq y, x-t / 2=y-h / 2$; then set $\alpha=y-x>0$, and proceed as in the previous case.

Case IV (ii) Let $x \neq y, x-t / 2>y-h / 2, x+t / 2<y+h / 2$. First suppose that $y<x$; put $\alpha=x-y$; so if $k=t+2 \alpha$ then $y+k / 2=x+t / 2$. Now since $[x-t / 2, x+t / 2] \subset[y-k / 2, y+k / 2]$, we have by Case II (ii) that

$$
0 \leq t^{m+1} \frac{\theta_{m+1}(x, t ; f)}{(m+1) !} \leq k^{m+1} \frac{\theta_{m+1}(y, k ; f)}{(m+1) !}
$$

and since $[y-k / 2, y+k / 2] \subset[y-h / 2, y+h / 2]$, by Case I

$$
0 \leq k^{m+1} \frac{\theta_{m+1}(y, k ; f)}{(m+1) !} \leq h^{m+1} \frac{\theta_{m+1}(y, h ; f)}{(m+1) !},
$$

which together give (6) with $m$ and $\phi$ replaced by $m+1$ and $f$. Finally if $x<y$ similar arguments hold and this completes the proof by induction of (ii).

To get similar results for the divided differences we need two lemmas.

Lemma 3.1. Let $g: \mathbb{R} \mapsto \mathbb{R}$ be $\mathcal{L}$-integrable in every finite interval, and let $g_{k}$ denote the $k$-fold repeated indefinite integral of $g$. Then for all $n \geq 2$, and for all $r, x, t \in \mathbb{R}$,

$$
\begin{aligned}
& \int_{r t}^{(r+1) t} \mathrm{~d} \xi_{1} \cdots \\
& \quad \int_{r t}^{(r+1) t}\left(g\left(x+\sum_{j=1}^{n-1} \xi_{j}+(1+r) t\right)-g\left(x+\Sigma_{j=1}^{n-1} \xi_{j}+r t\right)\right) \mathrm{d} \xi_{n-1} \\
& \quad=\sum_{i=0}^{n}(-1)^{n-i}\left(\begin{array}{c}
n \\
i
\end{array}\right) g_{n-1}(x+i t+n r t)
\end{aligned}
$$


Proof. It can be verified that the result is true for $n=2$. So suppose that it is true for $n=m \geq 2$. Then for all $r, x, t \in \mathbb{R}$ and for all $\xi_{m} \in \mathbb{R}$

$$
\begin{aligned}
& \int_{r t}^{(r+1) t} \mathrm{~d} \xi_{1} \cdots \\
& \int_{r t}^{(r+1) t}\left(g\left(x+\xi_{m}+\xi_{1}+\cdots+\xi_{m-1}+(1+r) t\right)-\right. \\
& \left.g\left(x+\xi_{m}+\xi_{1}+\cdots+\xi_{m-1}+r t\right)\right) \mathrm{d} \xi_{m-1} \\
& \quad=\sum_{i=0}^{m}(-1)^{m-i}\left(\begin{array}{c}
m \\
i
\end{array}\right) g_{m-1}\left(x+\xi_{m}+i t+m r t\right) .
\end{aligned}
$$

Integrating with respect to $\xi_{m}$,

$$
\begin{aligned}
& \int_{r t}^{(r+1) t} \mathrm{~d} \xi_{1} \cdots \\
& \int_{r t}^{(r+1) t}\left(g\left(x+\xi_{1}+\cdots+\xi_{m}+(1+r) t\right)-\right. \\
& \left.g\left(x+\xi_{1}+\cdots+\xi_{m}+r t\right)\right) \mathrm{d} \xi_{m} \\
& =\sum_{i=0}^{m}(-1)^{m-i}\left(\begin{array}{c}
m \\
i
\end{array}\right)\left(g_{m}(x+(i+1) t+(m+1) r t)\right. \\
& \left.\quad-g_{m}(x+i t+(m+1) r t)\right) \\
& =\sum_{j=1}^{m+1}(-1)^{m-j+1}\left(\begin{array}{c}
m \\
j-1
\end{array}\right) g_{m}(x+j t+(m+1) r t) \\
& =\sum_{j=0}^{m+1}(-1)^{m-j+1}\left(\begin{array}{c}
m+1 \\
j
\end{array}\right) g_{m}(x+j t+(m+1) r t),
\end{aligned}
$$

showing that the result is true for $n=m+1$. The proof is complete by induction.

Lemma 3.2. let $f: \mathbb{R} \mapsto \mathbb{R}$ be $n$-convex. If $x, y, r, t, h \in \mathbb{R}$ are such that 


$$
\begin{aligned}
& {[x+r t, x+(r+1) t] \subseteq[y+r h, y+(r+1) h] \text { then }} \\
& \quad 0 \leq \sum_{i=0}^{n}(-1)^{n-i}\left(\begin{array}{c}
n \\
i
\end{array}\right) f(x+i t+n r t) \leq \sum_{i=0}^{n}(-1)^{n-i}\left(\begin{array}{c}
n \\
i
\end{array}\right) f(y+i h+n r h) .
\end{aligned}
$$

Proof. Since $f$ is $n$-convex, $f^{(n-2)}$ exists and is convex, and so is an indefinite integral of $g=\left(f^{(n-2)}\right)_{+}^{\prime}$, which is an increasing function. Therefore, for every choice of $\xi_{1}, \ldots, \xi_{n-1}$,

$$
\begin{aligned}
0 \leq & g\left(x+\xi_{1}+\cdots+\xi_{n-1}+(r+1) t\right)-g\left(x+\xi_{1}+\cdots+\xi_{n-1}+r t\right) \\
& \leq g\left(y+\xi_{1}+\cdots+\xi_{n-1}+(r+1) h\right)-g\left(y+\xi_{1}+\cdots+\xi_{n-1}+r h\right)
\end{aligned}
$$

and so

$$
\begin{aligned}
0 \leq \int_{r t}^{(r+1) t}\left(g \left(x+\xi_{1}\right.\right. & \left.+\cdots+\xi_{n-1}+(r+1) t\right) \\
& \left.-g\left(x+\xi_{1}+\cdots+\xi_{n-1}+r t\right)\right) \mathrm{d} \xi_{\mathrm{n}-1} \\
\leq \int_{r h}^{(r+1) h}(g(y+ & \left.\xi_{1}+\cdots+\xi_{n-1}+(r+1) h\right) \\
& \left.-g\left(y+\xi_{1}+\cdots+\xi_{n-1}+r h\right)\right) \mathrm{d} \xi_{\mathrm{n}-1} .
\end{aligned}
$$

Integrating successively with respect to $\xi_{n-2}, \ldots, \xi_{1}$, and applying Lemma 3.1 the proof is complete.

Theorem 3.2. If $f: \mathbb{R} \mapsto \mathbb{R}$ is $n$-convex then for $t \geq 0$,

$$
0 \leq \Delta_{n}(x, t ; f) \leq \Delta_{n}(y, h ; f)
$$

whenever $\left[x-\frac{t}{2}, x+\frac{t}{2}\right] \subseteq\left[y-\frac{h}{2}, y+\frac{h}{2}\right]$;

$$
0 \leq \Delta_{n}^{*}(x, t ; f) \leq \Delta_{n}^{*}(y, h ; f)
$$

whenever $[x, x+t] \subseteq[y, y+h]$.

Proof. Put $r=-1 / 2$, and $r=0$ in Lemma 3.2 to get (i) and (ii) respectively. 


\section{Derivates, Approximate Derivates and Porosity Derivates of $n$-convex Functions}

We need the following porosity lemma due to Evans \& Humke.

Lemma 4.1. Let $g: \mathbb{R} \mapsto \mathbb{R}$ be an increasing function such that at a point $x_{0}, \underline{D}^{+} f\left(x_{0}\right)<r<s$. Then the set of points $Y^{s}=\left\{y ; \frac{f(y)-f\left(x_{0}\right)}{y-x_{0}}>s\right\}$ has porosity at least $1-r / s$ on the right at $x_{0}$. If $\bar{D}^{+} f\left(x_{0}\right)>s>r>0$ then the set of points $Y_{r}=\left\{y ; \frac{f(y)-f\left(x_{0}\right)}{y-x_{0}}<r\right\}$ has porosity at least $1-r / s$ on the right at $x_{0}$.

For a proof see [10, p.191].

Theorem 4.1. If $f: \mathbb{R} \mapsto \mathbb{R}$ is $n$-convex then at any point $x_{0}$

$$
\begin{array}{rlrl}
\text { (i) } & \bar{f}_{(n)}^{+}\left(x_{0}\right) & =\bar{f}_{(n), \mathrm{ap}}^{+}\left(x_{0}\right)=P \bar{f}_{(n)}^{+}\left(x_{0}\right) ; \\
\text { (ii }) & \bar{d}_{n} f\left(x_{0}\right)=\bar{d}_{n, \mathrm{ap}} f\left(x_{0}\right)=P \bar{d}_{n} f\left(x_{0}\right) ; \\
\text { (iii) } & \overline{R D}_{n} f\left(x_{0}\right)=\overline{R D}_{n, \mathrm{ap}} f\left(x_{0}\right)=P \overline{R D}_{n} f\left(x_{0}\right) ; \\
\text { (iv) } & \overline{R D}_{n}^{*+} f\left(x_{0}\right)=\overline{R D}_{n, \mathrm{ap}}^{*+} f\left(x_{0}\right)=P \overline{R D}_{n}^{*+} f\left(x_{0}\right) .
\end{array}
$$

Similar relations hold for the left upper derivates, and for the left and right lower derivates.

Proof. We first note that (cf. [10, p.155, Theorem 65.1]) if $g(t)$ is increasing for $t \geq 0$ then $\bar{D}^{+} g(0)=P \bar{D}^{+} g(0)$ where $\bar{D}^{+}$and $P \bar{D}^{+}$denote the right upper Dini derivate and porosity derivate respectively. Indeed, if $P \bar{D}^{+} g(0)<\bar{D}^{+} g(0)$ choose $P \bar{D}^{+} g(0)<r<s<\bar{D}^{+} g(0)$ when, by the second part of Lemma 4.1 the set $Y_{r}=\left\{y ; \frac{g(y)-g(0)}{y}<r\right\}$ has porosity at least $1-r / s$, and so $P \bar{D}^{+} g(0) \geq r$ which is a contradiction.

Now from Theorem 3.1(i) $t^{n} \gamma_{n}^{+}(\xi, t ; f)$ is a non-negative increasing function of $t, t \geq 0$. Hence if $g(t)=t\left(\gamma_{n}^{+}(\xi, t ; f)\right)^{1 / n}, t>0$ and $g(0)=0$ the function $g(t)$ is increasing for $t \geq 0$. Therefore from above $\bar{D}^{+} g(0)=P \bar{D}^{+} g(0)$. But

$$
D^{+} g(0)=\limsup _{t \rightarrow 0+} \frac{g(t)-g(0)}{t}=\limsup _{t \rightarrow 0+}\left(\gamma_{n}^{+}(\xi, t ; f)\right)^{1 / n}=\left(\bar{f}_{(n)}^{+}(\xi)\right)^{1 / n},
$$

and similarly $P \bar{D}^{+} g(0)=\left(P \bar{f}_{(n)}^{+}(\xi)\right)^{1 / n}$. Therefore $P \bar{f}_{(n)}^{+}(\xi)=\bar{f}_{(n)}^{+}(\xi)$, and since $P \bar{f}_{(n)}^{+}(\xi) \leq \bar{f}_{(n), \text { ap }}^{+}(\xi) \leq \bar{f}_{(n)}^{+}(\xi)$ the proof of (i) is complete. 
Applying Theorem 3.1(ii) and setting $g(t)=t\left(\theta_{n}(\xi, t ; f)^{1 / n}\right.$ for $t>0$ and $g(0)=0$, (ii) is proved as above.

To prove (iii) and (iv) note that by Theorem 3.2(i) and (ii) $\Delta_{n}(\xi, t ; f)$ and $\Delta_{n}^{*}(\xi, t ; f)$ are non-negative and increasing functions of $t$ for $t \geq 0$. Setting successively $g(t)=t\left(\frac{\Delta_{n}(\xi, t ; f)}{t^{n}}\right)^{1 / n}$, and $g(t)=t\left(\frac{\Delta_{n}^{*}(\xi, t ; f)}{t^{n}}\right)^{1 / n}$ for $t>0$ and $g(0)=0$ and applying the above argument the proof of (iii) and (iv) is completed as above.

The authors wish to thank the referee for his suggestions which shortened the proof of Theorem 4.1.

\section{References}

[1] A. M. Bruckner, Differentiation of Real Functions, Lecture Notes in Mathematics, Springer-Verlag, New York, 1978.

[2] A. M. Bruckner, \& B. S. Thomson, Porosity estimates for the Dini derivates, Real Anal. Exchange, 9(1983-1984), 508-538.

[3] P. S. Bullen, A criterion for n-convexity, Pacific J. Math., 36(1971), 8198.

[4] J. H. B. Kemperman, On the regularity of the generalized convex functions, Trans. Amer. Math. Soc., 135(1969),69-93.

[5] A. Khintchine, Recherches sur la structure des fonctions measurables, Fund. Math., 9(1927), 212-279 .

[6] P. Kostyrko, Approximate symmetric derivatives are uniformly closed, Real Anal. Exchange, 17(1991-1992), 652-656.

[7] N. C. Manna \& S. N. Mukhopadhyay, On approximate derivates and their properties, Acta Math. Acad. Sci. Hung., (3-4), 22(1971), 309-320.

[8] L. Misik, Über approximative derivierte Zahlen monotoner Funktionen, Czech. Math. J., 26(101) (1976), 579-583.

[9] T. Popoviciu, Les Fonctions Convexes, Acualités Sci. Indust., No. 992, Hermann, Paris, 1944.

[10] B. S. Thomson, Real Functions, Lecture Notes in Mathematics (1985), \#1170, Springer-Verlag. 
[11] B. S. Thomson, Symmetric Properties of Real Functions, Marcel Dekker Inc., 1994. 